\title{
Anticipatory Activity in Anterior Cingulate Cortex Can Be Independent of Conflict and Error Likelihood
}

\author{
Esther Aarts, ${ }^{1,2}$ Ardi Roelofs, ${ }^{1,2}$ and Miranda van Turennout ${ }^{1,3}$ \\ ${ }^{1}$ F. C. Donders Centre for Cognitive Neuroimaging, Radboud University Nijmegen, 6500 HB Nijmegen, The Netherlands, and ${ }^{2} \mathrm{Nijmegen} \mathrm{Institute} \mathrm{for}$ \\ Cognition and Information and ${ }^{3}$ Behavioural Science Institute, Radboud University Nijmegen, 6500 HE Nijmegen, The Netherlands
}

Previous studies have found no agreement on whether anticipatory activity in the anterior cingulate cortex (ACC) reflects upcoming conflict, error likelihood, or actual control adjustments. Using event-related functional magnetic resonance imaging, we investigated the nature of preparatory activity in the ACC. Informative cues told the participants whether an upcoming target would or would not involve conflict in a Stroop-like task. Uninformative cues provided no such information. Behavioral responses were faster after informative than after uninformative cues, indicating cue-based adjustments in control. ACC activity was larger after informative than uninformative cues, as would be expected if the ACC is involved in anticipatory control. Importantly, this activation in the ACC was observed for informative cues even when the information conveyed by the cue was that the upcoming target evokes no response conflict and has low error likelihood. This finding demonstrates that the ACC is involved in anticipatory control processes independent of upcoming response conflict or error likelihood. Moreover, the response of the ACC to the target stimuli was critically dependent on whether the cue was informative or not. ACC activity differed among target conditions after uninformative cues only, indicating ACC involvement in actual control adjustments. Together, these findings argue strongly for a role of the ACC in anticipatory control independent of anticipated conflict and error likelihood, and also show that such control can eliminate conflict-related ACC activity during target processing. Models of frontal cortex conflict-detection and conflict-resolution mechanisms require modification to include consideration of these anticipatory control properties of the ACC.

Key words: Stroop task; response conflict; preparation; cue-based adjustments; fMRI; neuroimaging

\section{Introduction}

Cognitive control refers to regulatory processes that ensure that our actions are in accordance with our goals. Neuroimaging experiments have shown that the anterior cingulate cortex (ACC) plays a role in cognitive control, together with other areas in frontal and parietal cortex (for review, see Picard and Strick, 1996; Bush et al., 2000; Miller, 2000; Paus, 2001). However, the exact function of the ACC in cognitive control is still a matter of debate. Some researchers have claimed that ACC activity reflects top-down regulation processes (Posner and Raichle, 1994; Roelofs and Hagoort, 2002; Swick and Turken, 2002; Roelofs, 2003; Dosenbach et al., 2006; Roelofs et al., 2006; Posner and Rothbart, 2007), whereas others have argued that ACC activity reflects the detection of competing response alternatives (Carter et al., 1999; MacDonald et al., 2000; Botvinick et al., 2001; Kerns et al., 2004). According to this latter conflict monitoring hypothesis, the occurrence of response conflict is signaled by the ACC and leads to

Received Sept. 26, 2007; revised March 11, 2008; accepted March 27, 2008.

This work was supported by a VICI Grant of the Netherlands Organization for Scientific Research to A.R. We thank Paul Gaalman for technical assistance, Marieke van der Linden for advice in data analysis, and Michael Coles for helpful comments on a previous version of this manuscript.

Correspondence should be addressed to Esther Aarts, F. C. Donders Centre for Cognitive Neuroimaging, Radboud University Nijmegen, P.0. Box 9101, 6500 HB Nijmegen, The Netherlands. E-mail: e.aarts@fcdonders.ru.nl. D01:10.1523/JNEUROSCI.4400-07.2008

Copyright $\odot 2008$ Society for Neuroscience $\quad 0270-6474 / 08 / 284671-08 \$ 15.00 / 0$ the recruitment of more cognitive control for subsequent performance executed by the lateral prefrontal cortex (LPFC).

Previously, Sohn et al. (2007) extended the role of the ACC as a response conflict monitor to include anticipatory conflict monitoring. Brown and Braver (2005) argued that the ACC predicts error likelihood, independent of response conflict. More generally, control adjustments can be made if environmental cues provide information about which type of target is coming and, as a consequence, about which control setting is most appropriate for processing the upcoming target (Logan and Zbrodoff, 1982; Logan, 1985; Gratton et al., 1992). However, these cues do not necessarily have to predict response conflict or error likelihood (Gratton et al., 1992). This raises the question whether anticipatory activity in the ACC may be obtained independent of upcoming conflict or error likelihood. We report a functional magnetic resonance imaging (fMRI) experiment that examined this issue.

Participants were informed about Stroop-like target conditions by means of symbolic cues, which were presented well before the imperative target on each trial (see Fig. 1). The symbolic cue indicated whether the upcoming Stroop target was congruent, incongruent, or neutral, or the cue provided no information about the upcoming condition. Earlier behavioral studies indicated that participants are able to process the cue and extract the information about the target condition it conveys and adjust their control accordingly (Logan and Zbrodoff, 1982; Gratton et al., 1992). 


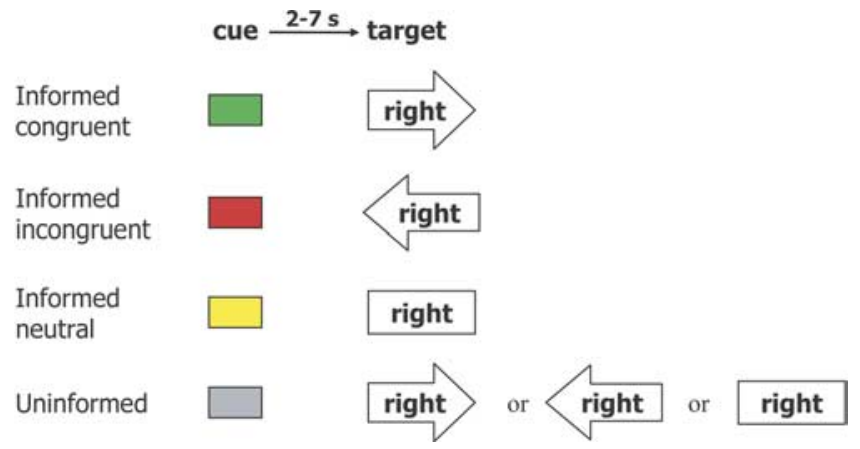

Figure 1. Experimental paradigm. Depicted are the informative and uninformative cues and examples of congruent, incongruent, and neutral targets with the word "right." Green cues were always followed by congruent targets, red cues were always followed by incongruent targets, and yellow cues were always followed by neutral targets. Gray cues could be followed by either one of the three target conditions and, hence, were uninformative. The task was to indicate the direction denoted by the word by pressing a left or right button.

If the ACC plays a role in anticipatory adjustments in control, ACC activity should be higher in response to informative cues than to uninformative cues. If the adjustments are independent of response conflict or error likelihood, enhanced ACC activity should be obtained for cues preceding congruent targets. Adjustments are expected in premotor cortex, where response rules are implemented (Wallis and Miller, 2003). Moreover, if the advance adjustments are successful, ACC activity should exhibit smaller differences among target conditions in response to targets after informative cues (when control was adjusted in advance) than after uninformative cues (when control was not adjusted in advance).

\section{Materials and Methods}

Subjects. Twelve neurologically healthy Dutch undergraduates ( 10 female and 2 male; mean age, 21.2 years; range, 18-24) participated in the experiment. All participants were right handed and native speakers of Dutch. They were compensated for participation and gave written informed consent in a manner approved by the Dutch Central Committee on Research Involving Human Subjects (CCMO).

Stimuli and paradigm. The participants were scanned while performing a manual arrow-word version of the Stroop task. As with color-word Stroop stimuli, responding in this task is usually slower on incongruent than on neutral trials and it is fastest on congruent trials (Baldo et al., 1998; Turken and Swick, 1999; Roelofs et al., 2006). The targets consisted of written words in arrows (see Fig. 1). The lines and letters of the targets were white on a black background. The arrows pointed to the left or to the right. The word in the arrow was the Dutch word for "right" ("rechts") or for "left" ("links"). Participants responded manually to the words of the Stroop-like targets by pressing a left or right button on a scanner-compatible button box. Participants were told to respond as quickly and accurately as possible with the left middle finger (for left response) and the left index finger (for right response). In the congruent target condition, the arrow and the word denoted the same direction (e.g., the word "right" in an arrow pointing to the right). In the incongruent target condition, the arrow and the word denoted a different direction (e.g., the word "left" in an arrow pointing to the right). In the neutral target condition, the targets consisted of words ("left" or "right") in rectangles without arrow points.

Every target was preceded by a cue (Fig. 1). The cue was a colored square giving either information about the upcoming target condition (informative cue) or giving no information (uninformative cue). The informative cues were $100 \%$ valid with green squares preceding congruent targets, red squares preceding incongruent targets, and yellow squares preceding neutral targets. The uninformative cues were gray squares, which could be followed by either one of the target types. Participants were told to pay explicit attention to the cues to let them be of help in processing the target. It was brought to the participants' attention that during congruent trials one could be helped by the nonrelevant dimension of the target (i.e., the arrow) and that one should not be distracted by the arrow in case of an incongruent trial (Logan and Zbrodoff, 1982). The experiment included 240 trials, consisting of 120 informative and 120 uninformative cues, and each type of trial containing 40 incongruent, 40 congruent, and 40 neutral targets. Informative and uninformative cues, as well as congruent, incongruent, and neutral targets were randomly intermixed.

The target followed the cue after a variable delay of 2-7 s. Similarly, a variable delay of 2-7 s was used between a target and the next cue. The jitter was calculated with a simulation of the blood oxygenation leveldependent (BOLD) response in SPM99 (Wellcome Department of Cognitive Neurology, London, UK). The variable delays enabled us to characterize the hemodynamic responses at a finer temporal resolution than the actual repetition time (Josephs et al., 1997) and thus allowed us to reliably distinguish the BOLD response to the cue from the BOLD response to the target [for a similar procedure, see Toni et al., (1999) and Mars et al. (2005)]. This calculation was repeated to generate a random sequence with optimal delays for every participant separately. Because the delay between cue and target could not be predicted, the participant needed to be ready to respond at any time. Cues and targets remained on the screen for $600 \mathrm{~ms}$.

Functional imaging. Whole-brain imaging was performed on a 3 Tesla MR scanner (Magnetom Trio; Siemens Medical Systems, Erlangen, Germany). Functional data were acquired using a gradient-echo echo-planar scanning sequence (repetition time, $2100 \mathrm{~ms}$; echo time, $30 \mathrm{~ms} ; 33$ axial slices; voxel size, $3.5 \times 3.5 \times 3.5 \mathrm{~mm}$; field of view, $224 \mathrm{~mm}$; flip angle, $70^{\circ}$ ). All functional images were acquired in a single run lasting $40 \mathrm{~min}$. Visual stimuli were projected on a screen and were viewed through a mirror attached to the head coil. After the acquisition of functional images, a high-resolution anatomical scan (T1-weighted magnetizationprepared rapid-acquisition gradient echo, 192 slices) was obtained.

Behavioral data analysis. The mean latencies of the correct manual responses and the error rates were analyzed using repeated-measures ANOVAs with the factors target condition (congruent, incongruent, neutral) and cue condition (informed, uninformed). All variables were tested within participants. Specific effects were tested with paired $t$ tests. An effect was called significant when $p<0.05$.

fMRI data analysis. fMRI data were analyzed with BrainVoyager QX (Brain Innovation, Maastricht, The Netherlands). Functional images were corrected for slice time acquisition (using sinc interpolation) and three-dimensional motion correction was performed to detect and correct for small head movements. Estimated translation and rotation parameters were inspected and never exceeded $3 \mathrm{~mm}$. Linear trend removal was performed and the signal was temporal high-pass filtered to remove low-frequency nonlinear drifts of three or fewer cycles per time course. Functional images were coregistered with the anatomical scan and transformed into Talairach coordinate space using the nine-parameter landmark method of Talairach and Tournoux (1988). Images were spatially smoothed with a full-width at half maximum Gaussian kernel of $6 \mathrm{~mm}$.

Statistical analyses were performed in the context of the general linear model, including the event types of interest: informative cues preceding congruent, incongruent, and neutral targets; uninformative cues preceding congruent, incongruent, and neutral targets; congruent, incongruent, and neutral informed targets; and congruent, incongruent, and neutral uninformed targets. Trials on which participants had made an error were put together as a separate event type of noninterest. Six motion parameters were included as event types of noninterest as well. The event types were modeled with a two gamma hemodynamic response function that was adjusted in such a way that it equaled the hemodynamic response function in SPM99 on the basis of which the jitter was calculated (see above). Random-effects group analyses were performed enabling generalization of the statistical inferences to the population level. A conjunction analysis with a standard "minimal $t$ statistic" approach (Nichols et al., 2005) was used with the contrasts (informative cues $>$ uninformative cues) $\cap$ (uninformed targets $>$ informed targets), to assess the effect of cue type on target processing. This conjunction analysis is equivalent to a logical AND of the contrasts at the voxel level. The statistical thresh- 
Table 1. Peak Talairach coordinates, cluster size, and peak $t$ values of regions showing an effect of advance information on the cue, and regions showing an effect of advance information on both the cue and the target for $p<0.001$ and a threshold of $50 \mathrm{~mm}^{3}$

\begin{tabular}{|c|c|c|c|c|c|c|c|c|c|c|c|}
\hline & \multicolumn{6}{|c|}{ Informative $>$ uninformative cues } & \multicolumn{5}{|c|}{ Informative $>$ uninformative cues AND uninformed $>$ informed targets } \\
\hline & $\mathrm{BA}$ & $x$ & $y$ & $z$ & Size $\left(\mathrm{mm}^{3}\right)$ & $t_{(11)}$ & $x$ & $y$ & $z$ & Size $\left(\mathrm{mm}^{3}\right)$ & $t_{(11)}$ \\
\hline \multicolumn{12}{|l|}{ Activations } \\
\hline \multirow[t]{2}{*}{$\mathrm{ACC}$} & 32 & -9 & 14 & 37 & 1762 & 6.77 & 9 & 14 & 40 & 540 & 5.87 \\
\hline & & -9 & -1 & 43 & 71 & 5.07 & -9 & 2 & 37 & 90 & 5.2 \\
\hline \multirow[t]{2}{*}{ DLPFC (MFG) } & 46 & -27 & 26 & 28 & 316 & 5.45 & & & & & \\
\hline & 9 & 30 & 32 & 34 & 901 & 9.16 & 30 & 32 & 31 & 192 & 6.99 \\
\hline Insula & 13 & 33 & 14 & 13 & 108 & 5.88 & & & & & \\
\hline SMA (MeFG) & 6 & -3 & -7 & 61 & 182 & 5.34 & & & & & \\
\hline \multirow[t]{2}{*}{ PMCd (SFG) } & 6 & -15 & -10 & 67 & 240 & 5.60 & -15 & -13 & 64 & 234 & 5.48 \\
\hline & & 12 & -7 & 64 & 216 & 5.35 & & & & & \\
\hline Precentral gyrus & 6 & 30 & -13 & 46 & 58 & 5.75 & & & & & \\
\hline \multirow[t]{2}{*}{$\mathrm{IPL}$} & 40 & -33 & -49 & 28 & 862 & 7.43 & -30 & -52 & 28 & 455 & 7.22 \\
\hline & & 54 & -46 & 19 & 196 & 6.44 & & & & & \\
\hline SPL & 7 & -30 & -58 & 55 & 178 & 5.59 & & & & & \\
\hline Angular gyrus & 39 & -27 & -61 & 22 & 56 & 6.86 & & & & & \\
\hline \multirow[t]{4}{*}{ Precuneus } & 7 & -15 & -58 & 40 & 65 & 5.24 & -6 & -64 & 55 & 370 & 5.54 \\
\hline & & -15 & -67 & 52 & 1223 & 7.37 & -15 & -67 & 52 & 120 & 5.51 \\
\hline & & & & & & & -9 & -73 & 40 & 62 & 4.91 \\
\hline & & 12 & -73 & 46 & 1545 & 7.73 & 12 & -76 & 43 & 1065 & 6.46 \\
\hline Parahip. gyrus & 19 & 18 & -46 & -5 & 61 & 5.39 & & & & & \\
\hline \multirow[t]{2}{*}{ Lingual gyrus } & 18 & -9 & -94 & -8 & 58 & 5.28 & -6 & -61 & -5 & 142 & 5.86 \\
\hline & & 0 & -76 & 1 & 409 & 5.96 & & & & & \\
\hline \multirow[t]{2}{*}{ Cuneus } & $18 / 17$ & 3 & -88 & 19 & 134 & 4.93 & 0 & -82 & 13 & 158 & 5.69 \\
\hline & 18 & 15 & -91 & 7 & 75 & 5.72 & & & & & \\
\hline \multirow[t]{2}{*}{ Dorsal striatum } & & -21 & 14 & 10 & 918 & 6.64 & -21 & 14 & 10 & 497 & 6.02 \\
\hline & & 18 & 11 & 10 & 1180 & 8.28 & 21 & 11 & -2 & 519 & 6.55 \\
\hline Thalamus & & -15 & -7 & 13 & 775 & 6.79 & -15 & -7 & 10 & 76 & 5.34 \\
\hline \multirow[t]{3}{*}{ Cerebellum } & & -36 & -52 & -29 & 1480 & 6.96 & -36 & -49 & -29 & 443 & 6.14 \\
\hline & & & & & & & -24 & -61 & -17 & 51 & 5.21 \\
\hline & & 42 & -49 & -29 & 4743 & 8.36 & 21 & -67 & -20 & 2350 & 7.82 \\
\hline \multicolumn{12}{|l|}{ Deactivations } \\
\hline MeFG & 9 & -3 & 47 & 28 & 98 & -5.11 & & & & & \\
\hline \multirow[t]{2}{*}{ Subgenual area } & 25 & -9 & 29 & -5 & 486 & -6.23 & & & & & \\
\hline & & 0 & 20 & -14 & 55 & -6.03 & & & & & \\
\hline
\end{tabular}

MFG, Middle frontal gyrus; MeFG, medial frontal gyrus; PMCd, dorsal premotor cortex; SFG, superior frontal gyrus; IPL, inferior parietal lobule; SPL, superior parietal lobule; Parahip., parahippocampal.

old for the group analyses was set at $p<0.001$ at the voxel level with a minimum cluster size of $50 \mathrm{~mm}^{3} / 14$ original voxels (Forman et al., 1995), uncorrected for multiple comparisons.

To investigate differential effects of cue information in the ACC, we obtained subject-averaged $\beta$ weights (i.e., regression coefficients) for all cue conditions as indices of effect size for all voxels in the functionally defined region of the ACC showing an effect of informative cues versus uninformative cues in the random effects group analysis. To investigate the effect of cue information on target-related effects in the ACC, subjectaveraged $\beta$ weights were extracted for all target conditions from ACC voxels showing an effect of informative cues versus uninformative cues in the random effects group analysis. In addition, to ensure that the observed effects were the same regardless of the contrast used to select the $\beta$ weights, subject averaged $\beta$ weights were extracted for each event type from ACC voxels showing an effect of incongruent versus congruent targets in the uninformative condition. Regionally averaged $\beta$ weights were analyzed in repeated-measurement ANOVAs. Specific effects were tested by applying paired $t$ contrasts to the $\beta$ weights obtained for the different event types. The regional-specific time courses were standardized, so that $\beta$ weights reflected the BOLD response amplitude of one condition relative to the variability of the signal. An effect was called significant when $p<0.05$.

Premotor cortex activity should reflect the operation of control in response to informative cues. Therefore, we expected a positive correlation between ACC and premotor activity. To test these predictions, we computed Pearson correlations between the $\beta$ weights in the ACC and the regions differentially activated on the cues in Table 1 . We mention only correlations that were significant for all three separate cue conditions and report $p$ values on a Bonferroni corrected $\alpha$ level $(p<0.002)$ across subjects and cue conditions $(n=36)$. Furthermore, we tested for

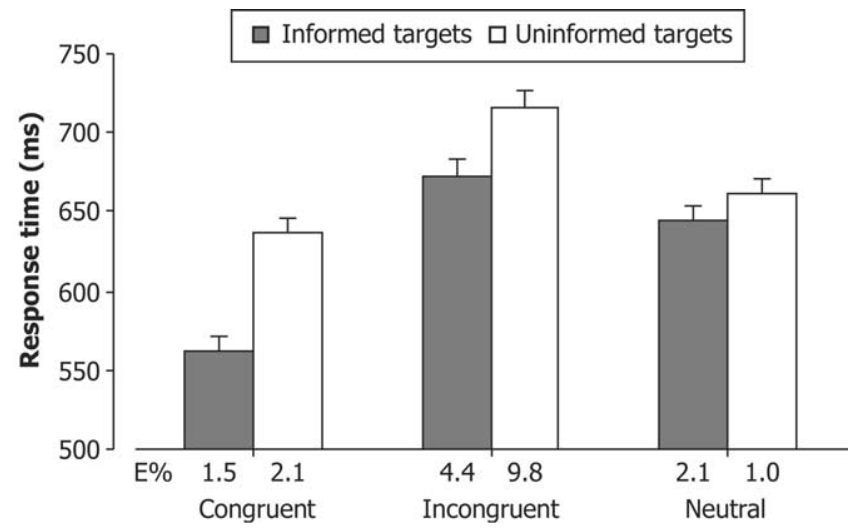

Figure 2. Behavioral results. Mean response times and error rates (E\%) of congruent, neutral, and incongruent target conditions preceded by informative and uninformative cues. Error bars represent SEM.

the significance of the difference between (dependent) correlations (Chen and Popovich, 2002).

\section{Results}

\section{Behavioral data}

Analysis of the reaction-time data (Fig. 2) showed a main effect of target condition $\left(F_{(2,22)}=69.51 ; p<0.001\right)$ and a main effect of cue condition $\left(F_{(1,11)}=45.36 ; p<0.001\right)$. The interaction between cue condition and target condition was significant 
$\left(F_{(2,22)}=7.48 ; p=0.003\right)$. A similar pattern was observed for the errors. The analysis yielded a main effect for target condition $\left(F_{(2,22)}=7.66 ; p=0.003\right)$, a marginally significant main effect for cue condition $\left(F_{(1,11)}=4.17 ; p=0.066\right)$, and a significant interaction between cue condition and target condition $\left(F_{(2,22)}=6.19\right.$; $p=0.007$ ).

Reaction times were slower in the incongruent than in the neutral condition $\left(t_{(11)}=5.33 ; p<0.000\right)$, and fastest in the congruent condition (compared with neutral, $\left.t_{(11)}=8.97 ; p<0.000\right)$. Most errors were made in the incongruent condition (compared with neutral, $t_{(11)}=2.85 ; p=$ 0.008 , one-tailed; compared with congruent, $t_{(11)}=2.98 ; p=0.007$, one-tailed), whereas the neutral and congruent condition did not differ $\left(t_{(11)}=-0.33 ; p=\right.$ 0.742 ). Thus, conflict and error likelihood were higher in the incongruent condition than in the other conditions.

The difference in response times between congruent and neutral targets (i.e., the facilitation effect) was larger after informative cues $(83 \mathrm{~ms})$ than after uninformative cues (25 ms) (Fig. 2) $\left(t_{(11)}=4.47\right.$; $p<0.001$, one-tailed). The difference between incongruent and neutral targets (i.e., the interference effect) was marginally smaller after informative cues $(27 \mathrm{~ms})$ than after uninformative cues $(56 \mathrm{~ms})\left(t_{(11)}=\right.$ 1.52; $p=0.079$, one-tailed). In other words, advance information tripled the Stroop-like facilitation effect and halved the interference effect.

A cue limited the target types to two. It is therefore possible that the speed up of responses to informed targets was caused by exact target expectation, which may be correct on half the trials. If so, the speed up should be present on about half the trials only. To test this, we classified the response times (RTs) from each condition as below or above the median condition RT, and tested for an interaction between cue condition and relative speed. There were no such interactions for the congruent $\left(F_{(1,11)}\right.$ $=1.64 ; p=0.227)$, incongruent $\left(F_{(1,11)}=1.90 ; p=0.195\right)$, and neutral trials $\left(F_{(1,11)}<1\right)$. These results exclude that the cuebased anticipatory effects were caused by exact target expectation.

\section{Neuroimaging data}

Comparing fMRI responses to informative cues with fMRI responses to uninformative cues revealed strong activity in a network of brain regions, including the ACC (Table 1).

In addition, a conjunction analysis of the contrasts [informative cue $>$ uninformative cue] and [uninformed target $>$ informed target] revealed that the ACC and other brain regions (listed in Table 1) showed more activity for informative than for uninformative cues, and subsequently, reduced activity for informed compared with uninformed targets.

To further examine ACC responses to cue information, subject-averaged $\beta$ weights were extracted for all voxels in the
ACC region showing increased activity for informative compared with uninformative cues (Fig. $3 a$ ). This was done for cues informative of incongruent, congruent, and neutral targets. Pairwise comparisons of these $\beta$ weights showed that ACC activity was significantly larger for cues informative of incongruent and congruent targets compared with cues informative of neutral targets $t_{(11)}=2.43, p=0.017$, one-tailed; and $t_{(11)}=2.74, p=0.01$, one-tailed, respectively) (Fig. $3 b$ ). Importantly, cues informative of congruent and incongruent targets elicited similar ACC activity $\left(t_{(11)}=-0.17 ; p=0.869\right)$ (Fig. $\left.3 b\right)$.

Comparing $\mathrm{fMRI}$ responses to incongruent and congruent targets revealed increased activity in brain regions listed in Table 2. After uninformative cues, a standard Stroop effect was observed in a network of regions including the ACC. After informative cues, only the left dorsolateral PFC (DLPFC) and left ventrolateral prefrontal cortex were more active for incongruent than for congruent targets. To further investigate this effect of cue information on target processing in the ACC, subject-averaged $\beta$ weights were extracted from the ACC region responding to informative cues (Fig. $3 a$ ), separately for each of the target conditions. As can be seen in Figure 3, $c$ and $d$, ACC activity was significantly reduced for targets preceded by an informative cue compared with targets preceded by an 
Table 2. Peak Talairach coordinates, cluster size, and peak $t$ values of regions showing more activity for incongruent than congruent targets for $p<0.001$ and a threshold of $50 \mathrm{~mm}^{3}$

\begin{tabular}{|c|c|c|c|c|c|c|}
\hline & $B A$ & $x$ & $y$ & $z$ & Size $\left(\mathrm{mm}^{3}\right)$ & $t_{(11)}$ \\
\hline \multicolumn{7}{|l|}{ Uninformed targets } \\
\hline $\mathrm{ACC}$ & 24 & 6 & 8 & 34 & 1223 & 6.24 \\
\hline Insula & & -30 & 8 & 1 & 205 & 5.91 \\
\hline Precentral gyrus & 6 & 36 & -1 & 34 & 716 & 6.16 \\
\hline SMA (MeFG) & 6 & -6 & -4 & 58 & 321 & 6.03 \\
\hline \multirow[t]{2}{*}{ MFG } & 6 & -24 & -10 & 55 & 1125 & 8.07 \\
\hline & & 21 & -7 & 58 & 118 & 5.47 \\
\hline$C G$ & 23 & 3 & -22 & 28 & 1325 & 6.57 \\
\hline \multirow[t]{5}{*}{ IPL } & 40 & -45 & -43 & 52 & 88 & 9.23 \\
\hline & & -36 & -43 & 37 & 156 & 6.24 \\
\hline & & -54 & -49 & 25 & 63 & 5.37 \\
\hline & & -60 & -31 & 34 & 99 & 5.19 \\
\hline & & 60 & -31 & 25 & 502 & 5.92 \\
\hline \multirow[t]{3}{*}{ SPL } & 7 & -15 & -64 & 52 & 3091 & 8.96 \\
\hline & & 15 & -67 & 55 & 193 & 5.55 \\
\hline & & 24 & -52 & 55 & 72 & 4.97 \\
\hline \multirow[t]{3}{*}{ Precuneus } & 7 & -24 & -73 & 43 & 104 & 4.88 \\
\hline & & 9 & -52 & 67 & 51 & 6.20 \\
\hline & 31 & 12 & -64 & 28 & 2772 & 7.08 \\
\hline Angular gyrus & 39 & 30 & -55 & 34 & 153 & 5.65 \\
\hline \multirow[t]{2}{*}{ MTG } & 39 & 45 & -64 & 10 & 56 & 5.54 \\
\hline & 21 & -60 & -49 & 10 & 152 & 5.80 \\
\hline \multirow[t]{3}{*}{ SOG } & 19 & -27 & -67 & 25 & 253 & 6.60 \\
\hline & & -24 & -76 & 34 & 66 & 5.50 \\
\hline & & 33 & -73 & 28 & 162 & 5.95 \\
\hline Cuneus & 19 & -3 & -73 & 28 & 69 & 5.28 \\
\hline \multirow[t]{3}{*}{ Lingual gyrus } & 18 & -9 & -82 & -11 & 396 & 6.12 \\
\hline & & 18 & -97 & -8 & 110 & 8.57 \\
\hline & & 3 & -85 & 1 & 287 & 6.08 \\
\hline \multirow[t]{2}{*}{ Thalamus } & & -12 & -19 & 4 & 352 & 5.76 \\
\hline & & 15 & -10 & 4 & 87 & 6.00 \\
\hline Cerebellum & & 21 & -55 & -42 & 61 & 5.71 \\
\hline Caudate & & 9 & 5 & 4 & 61 & 5.43 \\
\hline \multicolumn{7}{|l|}{ Informed targets } \\
\hline DLPFC (MFG) & 46 & -39 & 41 & 16 & 73 & 6.69 \\
\hline VLPFC (IFG) & 46 & -48 & 38 & 7 & 93 & 5.25 \\
\hline
\end{tabular}

MeFG, Medial frontal gyrus; MFG, middle frontal gyrus; CG, cingulate gyrus; IPL, inferior parietal lobule; SPL, superior parietal lobule; MTG, middle tempora gyrus; SOG, superior occipital gyrus; VLPFC, ventrolateral prefrontal cortex; IFG, inferior frontal gyrus.

uninformative cue $\left(t_{(11)}=3.89 ; p=0.002\right)$. Uninformed targets elicited a normal Stroop-like pattern of activity with larger responses for incongruent than for congruent targets $\left(t_{(11)}=4.77 ; p<0.001\right.$, one-tailed $)$, and reduced responses for congruent compared with neutral targets $\left(t_{(11)}=3.84 ; p=\right.$ 0.002 , one-tailed). Interestingly, for informed targets, ACC responses did not differ between target conditions (incongruent $>$ congruent, $t_{(11)}=-0.28, p=0.389$, one-tailed; congruent $<$ neutral, $t_{(11)}=-0.52, p=0.307$, one-tailed). This result shows that although a standard Stroop-like effect was obtained in the ACC for uninformed targets, this effect disappeared when a target was preceded by an informative cue.

To examine whether a similar effect of cue information on target processing was present in ACC voxels showing a strong Stroop effect for uninformed targets, subject-averaged $\beta$ weights were obtained for all voxels in the ACC showing larger responses for incongruent compared with congruent targets preceded by uninformative cues. Paired comparisons of the cue-related $\beta$ weights showed that ACC responses were larger for informative than for uninformative cues $\left(t_{(11)}=4.56\right.$; $p<$ 0.001 , one-tailed). More specifically, ACC responses were larger for cues informative of incongruent and congruent targets compared with cues informative of neutral targets $\left(t_{(11)}=\right.$
$2.29, p=0.022$, one-tailed; and $t_{(11)}=$ 2.46, $p=0.016$, one-tailed, respectively). Again, no difference in ACC activity was observed for cues informative of incongruent and congruent targets $\left(t_{(11)}=-0.1 ; p=0.911\right)$. Paired comparisons of target-related $\beta$ weights obtained in this ACC region showed significantly less ACC activity for targets preceded by an informative cue than for targets preceded by an uninformative cue $\left(t_{(11)}=3.1 ; p=0.005\right)$. Importantly, although $\beta$ weights were obtained from those ACC voxels responding more strongly to incongruent targets than to congruent targets in the uninformed condition, no such Stroop-like effects were found for targets that were preceded by an informative cue. For informed targets, ACC responses did not differ between target conditions (incongruent $>$ congruent, $t_{(11)}=-0.17, p=$ 0.43 , one-tailed; congruent $<$ neutral, $t_{(11)}=-0.36, p=0.365$, one-tailed). Thus, the difference between incongruent and congruent targets (the Stroop effect) in the ACC was larger after uninformative cues than after informative cues $\left(t_{(11)}=1.97 ; p=0.037\right.$, one-tailed $)$.

Additional analyses showed that cuebased activity in the ACC was positively correlated for all three informative cue conditions separately with activity in the dorsal premotor cortex contralateral to the response hand [right superior frontal gyrus, Brodmann's area (BA) 6; overall $\left.r=0.69 ; t_{(34)}=5.54 ; p<0.001\right]$ and the supplementary motor area (SMA; BA 6; overall $\left.r=0.75 ; t_{(34)}=6.65 ; p<0.001\right)$. There were no such correlations between cue-based ACC activity and any of the other regions listed in Table 1, except the cerebellum ipsilateral to the response hand (overall $r=0.74 ; t_{(34)}=6.47 ; p<0.001$ ). Cue-related activity in left DLPFC, although often coactivated with the ACC (Koski and Paus, 2000) and similarly coactivated in the present study (Table 1), did not show a significant correlation with cue-related ACC activity (overall $r=0.22 ; p>0.1$ ). The correlations between activity in the ACC and the right dorsal premotor cortex and between the ACC and the SMA were significantly greater than the correlation between activity in the ACC and the left DLPFC $\left(t_{(33)}=3.39, p<0.01\right.$; and $t_{(33)}=$ $3.51, p<0.01$, respectively). These results provide evidence that activity in premotor cortex indexes the operation of control in response to informative cues, as predicted.

\section{Discussion}

In the present study, we investigated the role of the ACC in preparatory adjustments in control by using symbolic cues that informed participants about upcoming Stroop-like target conditions. Our fMRI data show that the ACC was directly involved in symbolically driven adjustments in control. ACC activity was significantly enhanced for informative cues compared with uninformative cues. This finding is consistent with previous brain- 
imaging reports showing enhanced ACC activity in response to informative task or stimulus cues in a variety of task situations (Murtha et al., 1996; Luks et al., 2002; Weissman et al., 2005; Dosenbach et al., 2006; Parris et al., 2007). Previously, electrophysiological recordings from monkey cortex provided evidence for task-related preparatory activation in ACC neurons (Johnston et al., 2007).

Sohn et al. (2007) also demonstrated that the ACC is active during anticipatory preparation. Similar to our results, in their study, the ACC was only differentially active between low- and high-conflict targets when there was no opportunity to prepare. However, Sohn et al. (2007) claim that the anticipatory activity in the ACC is critically dependent on upcoming response conflict. In their view, the ACC monitors conflict regardless of whether the source is online or anticipatory. In contrast to this view, our data show that anticipatory ACC activity can be independent of response conflict. In the present experiment, the ACC was equally active for cues indicating an upcoming incongruent target and cues indicating an upcoming congruent target, although with congruent targets there are no competing response alternatives.

Brown and Braver (2005) presented data that also challenged the claim that anticipatory activity in the ACC reflects conflict monitoring. They showed that the ACC can be active in trials in which there is no response conflict. However, they argued that the ACC predicts error likelihood (Magno et al., 2006) (but see Nieuwenhuis et al., 2007). In contrast to this view, we found that ACC activity was enhanced for informative cues preceding congruent as well as incongruent targets, although participants made significantly more errors in the incongruent condition than in the congruent one. Thus, although the error likelihood was higher for incongruent trials than for congruent trials, no difference in ACC activity was obtained for the cues. The independence of ACC activity from error likelihood in the present study is also evident from comparing congruent and neutral trials in which the amount of response conflict is the same (absent in both cases) and the error likelihood is the same (see Results, Behavioral data). Despite similar conflict and error likelihood levels, the ACC was more active for cues preceding congruent trials than for cues preceding neutral trials. This clearly shows that the involvement of the ACC in preparatory control is not restricted to conflict or high error likelihood situations.

In a previous study, Luks et al. (2007) used cues to inform participants about upcoming Eriksen flanker conditions. However, unlike what we observed, Luks et al. (2007) did not find anticipatory ACC activity in response to the informative cues. The authors argued that the flanker task involves stimulus conflict rather than response conflict (but see Sanders and Lamers, 2002). Luks et al. (2007) expected to find ACC activity in preparation for response conflict. However, our data show that ACC activity is independent of upcoming response conflict. Importantly, in contrast to our findings, Luks et al. (2007) obtained no behavioral evidence for adjustments in control based on the cues. That is, the flanker effect in the RTs did not differ between informative and uninformative cues. Thus, it seems that Luks et al. (2007) did not find cue-related ACC activity simply because their participants did not adjust control in response to the cues.

If ACC activity in response to informative cues is independent of response conflict or error likelihood, what does it reflect? The behavioral data give some clues about what is happening on the cues. For both informed and uninformed targets we observed the normal Stroop pattern: participants were slowest on the incongruent trials and fastest on the congruent trials (Baldo et al., 1998; Turken and Swick, 1999; Roelofs et al., 2006). However, partici- pants were faster after informative cues than after uninformative cues. Moreover, the interference effect was numerically smaller after informative cues than after uninformative cues. Also, the facilitation effect was much larger after informative cues than after uninformative cues. A similar cueing benefit was observed in other studies using cues to inform participants about the upcoming target condition (Logan and Zbrodoff, 1982; Gratton et al., 1992). In these studies, the largest cueing benefit was obtained for the congruent targets, as was the case in the present study. The cues in these previous studies and in the present experiment may elicit control adjustments aiming at optimal processing of the upcoming target (Gratton et al., 1992). An informative cue preceding an incongruent target might encourage participants to strengthen the connections between the words and their responses, because the irrelevant arrows elicit the wrong response. However, an informative cue preceding a congruent target might encourage participants to strengthen the connections between the arrows and the corresponding responses, because the irrelevant arrows also elicit the correct response. Overall, our behavioral results show that control adjustments are made on the basis of symbolic cues.

Our imaging results provide evidence for a role of the ACC in these preparatory adjustments in cognitive control. If the ACC is involved in adjusting control settings such that they are most appropriate for responding to the upcoming targets, then ACC activity should be enhanced in response to informative cues preceding both types of targets. This is indeed what we observed. In case of an upcoming neutral stimulus, control adjustments can be less because there is no incongruent arrow to ignore or congruent arrow to exploit in responding. This explains the finding of less ACC activity for informative cues preceding neutral targets compared with informative cues preceding congruent and incongruent targets. Moreover, the advance adjustments appeared to be successful as is evident from the reaction time and imaging data. That is, the response of the ACC to the target stimuli was critically dependent on whether the cue was informative or not. After informative cues, there were no differences in ACC activity among target conditions, whereas in absence of advance information, a normal Stroop pattern was observed.

Previous studies have examined consequences of control adjustments by looking at the effects of control on behavioral measures and task-selective brain regions (Egner and Hirsch, 2005; Yeung et al., 2006). Specifically, effective connectivity studies provided evidence for a function of the ACC in regulating or top-down modulation of activity in modality-specific sensory areas (Crottaz-Herbette and Menon, 2006), the amygdala (Etkin et al., 2006), and the caudal cingulate (motor) zone (Fan et al., 2007). The effective connectivity from rostral ACC to caudal cingulate zone was modulated by conflict. The caudal cingulate activation extended into the SMA, a region that was functionally coupled to the rostral ACC in the present study. The present finding that ACC activity only correlated with activity in premotor cortex/SMA after informative cues, and not with activity in any other cortical area including the left DLPFC, similarly suggests that the ACC has a regulative role itself (Johnston et al., 2007). We are aware of the limitations of correlation analyses regarding directionality interpretations. Still, given the findings of Fan et al. (2007), it seems plausible to assume that the ACC exerts an influence over premotor cortex/SMA rather than the other way around.

Our data suggest that the ACC is actively involved in setting control parameters. This idea fits with reinforcement learning theories, according to which the ACC uses positive (reward) and 
negative (e.g., error) information to identify and select appropriate behaviors (Holroyd and Coles, 2002; Walton et al., 2003; Rushworth et al., 2004; Williams et al., 2004; Amiez et al., 2006; Somerville et al., 2006). Our data suggest that, in addition to rewards and errors, symbolic cues can be used to inform the ACC that it should adjust control settings. A role for the ACC in adjusting control also fits with previous neuroimaging evidence that the ACC is activated in decision making when the freedom of choice increases (Walton et al., 2004; Forstmann et al., 2006) or when a task is novel or difficult and that activity diminishes after practice (Raichle et al., 1994; Bush et al., 1998; Milham et al., 2003). These findings can readily be explained in terms of control adjustments. Appropriate behaviors are more easily selected after extensive training/practice or when explicitly instructed; hence, control adjustments can be less.

To conclude, our results demonstrate that the ACC is involved in preparatory adjustments in control, driven by symbolic cues and independent of anticipated response conflict and error likelihood. When control can be adjusted in advance, the ACC is no longer involved in resolving Stroop-like conflict evoked by the target. The present findings argue strongly for a role of the ACC in actual control adjustments. Models of frontal cortex conflictdetection and conflict-resolution mechanisms will require modification to include consideration of these anticipatory control properties of the ACC.

\section{References}

Amiez C, Joseph JP, Procyk E (2006) Reward encoding in the monkey anterior cingulate cortex. Cereb Cortex 16:1040-1055.

Baldo JV, Shimamura AP, Prinzmetal W (1998) Mapping symbols to response modalities: interference effects on Stroop-like tasks. Percept Psychophys 60:427-437.

Botvinick M, Braver TS, Barch DM, Carter CS, Cohen JD (2001) Conflict monitoring and cognitive control. Psychol Rev 108:624-652.

Brown JW, Braver TS (2005) Learned predictions of error likelihood in the anterior cingulate cortex. Science 307:1118-1121.

Bush G, Whalen PJ, Rosen BR, Jenike MA, McInerney SC, Rauch SL (1998) The counting Stroop: an interference task specialized for functional neuroimaging-validation study with functional MRI. Hum Brain Mapp 6:270-282.

Bush G, Luu P, Posner MI (2000) Cognitive and emotional influences in anterior cingulate cortex. Trends Cogn Sci 4:215-222.

Carter CS, Botvinick MM, Cohen JD (1999) The contribution of the anterior cingulate cortex to executive processes in cognition. Rev Neurosci 10:49-57.

Chen PY, Popovich PM (2002) Correlation: parametric and nonparametric measures. Thousand Oaks, CA: Sage.

Crottaz-Herbette S, Menon V (2006) Where and when the anterior cingulate cortex modulates attentional response: combined fMRI and ERP evidence. J Cogn Neurosci 18:766-780.

Dosenbach NU, Visscher KM, Palmer ED, Miezin FM, Wenger KK, Kang HC, Burgund ED, Grimes AL, Schlaggar BL, Petersen SE (2006) A core system for the implementation of task sets. Neuron 50:799-812.

Egner T, Hirsch J (2005) Cognitive control mechanisms resolve conflict through cortical amplification of task-relevant information. Nat Neurosci 8:1784-1790.

Etkin A, Egner T, Peraza DM, Kandel ER, Hirsch J (2006) Resolving emotional conflict: a role for the rostral anterior cingulate cortex in modulating activity in the amygdala. Neuron 51:871-882.

Fan J, Hof PR, Guise KG, Fossella JA, Posner MI (2007) The functional integration of the anterior cingulate cortex during conflict processing. Cereb Cortex 18:796-805.

Forman SD, Cohen JD, Fitzgerald M, Eddy WF, Mintun MA, Noll DC (1995) Improved assessment of significant activation in functional magnetic resonance imaging (fMRI): use of a cluster-size threshold. Magn Reson Med 33:636-647.

Forstmann BU, Brass M, Koch I, von Cramon DY (2006) Voluntary selection of task sets revealed by functional magnetic resonance imaging. J Cogn Neurosci 18:388-398.
Gratton G, Coles MG, Donchin E (1992) Optimizing the use of information: strategic control of activation of responses. J Exp Psychol Gen 121:480-506.

Holroyd CB, Coles MG (2002) The neural basis of human error processing: reinforcement learning, dopamine, and the error-related negativity. Psychol Rev 109:679-709.

Johnston K, Levin HM, Koval MJ, Everling S (2007) Top-down controlsignal dynamics in anterior cingulate and prefrontal cortex neurons following task switching. Neuron 53:453-462.

Josephs O, Turner R, Friston K (1997) Event-related fMRI. Hum Brain Mapp 5:243-248.

Kerns JG, Cohen JD, MacDonald III AW, Cho RY, Stenger VA, Carter CS (2004) Anterior cingulate conflict monitoring and adjustments in control. Science 303:1023-1026.

Koski L, Paus T (2000) Functional connectivity of the anterior cingulate cortex within the human frontal lobe: a brain-mapping meta-analysis. Exp Brain Res 133:55-65.

Logan GD (1985) Executive control of thought and action. Acta Psychol (Amst) 60:193-210.

Logan GD, Zbrodoff NJ (1982) Constraints on strategy construction in a speeded discrimination task. J Exp Psychol Hum Percept Perform 8:502-520.

Luks TL, Simpson GV, Feiwell RJ, Miller WL (2002) Evidence for anterior cingulate cortex involvement in monitoring preparatory attentional set. NeuroImage 17:792-802.

Luks TL, Simpson GV, Dale CL, Hough MG (2007) Preparatory allocation of attention and adjustments in conflict processing. NeuroImage 35:949-958.

MacDonald III AW, Cohen JD, Stenger VA, Carter CS (2000) Dissociating the role of the dorsolateral prefrontal and anterior cingulate cortex in cognitive control. Science 288:1835-1838.

Magno E, Foxe JJ, Molholm S, Robertson IH, Garavan H (2006) The anterior cingulate and error avoidance. J Neurosci 26:4769-4773.

Mars RB, Coles MG, Grol MJ, Holroyd CB, Nieuwenhuis S, Hulstijn W, Toni I (2005) Neural dynamics of error processing in medial frontal cortex. NeuroImage 28:1007-1013.

Milham MP, Banich MT, Claus ED, Cohen NJ (2003) Practice-related effects demonstrate complementary roles of anterior cingulate and prefrontal cortices in attentional control. NeuroImage 18:483-493.

Miller EK (2000) The prefrontal cortex and cognitive control. Nat Rev Neurosci 1:59-65.

Murtha S, Chertkow H, Beauregard M, Dixon R, Evans AC (1996) Anticipation causes increased blood flow to the anterior cingulate cortex. Hum Brain Mapp 4:103-112.

Nichols T, Brett M, Andersson J, Wager T, Poline JB (2005) Valid conjunction inference with the minimum statistic. NeuroImage 25:653-660.

Nieuwenhuis S, Schweizer TS, Mars RB, Botvinick MM, Hajcak G (2007) Error-likelihood prediction in the medial frontal cortex: a critical evaluation. Cereb Cortex 17:1570-1581.

Parris BA, Thai NJ, Benattayallah A, Summers IR, Hodgson TL (2007) The role of the lateral prefrontal cortex and anterior cingulate in stimulusresponse association reversals. J Cogn Neurosci 19:13-24.

Paus T (2001) Primate anterior cingulate cortex: where motor control, drive and cognition interface. Nat Rev Neurosci 2:417-424.

Picard N, Strick PL (1996) Motor areas of the medial wall: a review of their location and functional activation. Cereb Cortex 6:342-353.

Posner MI, Raichle ME (1994) Images of mind. New York: W. H. Freeman.

Posner MI, Rothbart MK (2007) Res on attention networks as a model for the integration of psychological science. Annu Rev Psychol 58:1-23.

Raichle ME, Fiez JA, Videen TO, MacLeod AM, Pardo JV, Fox PT, Petersen SE (1994) Practice-related changes in human brain functional anatomy during nonmotor learning. Cereb Cortex 4:8-26.

Roelofs A (2003) Goal-referenced selection of verbal action: modeling attentional control in the Stroop task. Psychol Rev 110:88-125.

Roelofs A, Hagoort P (2002) Control of language use: cognitive modeling of the hemodynamics of Stroop task performance. Brain Res Cogn Brain Res 15:85-97.

Roelofs A, van Turennout M, Coles MG (2006) Anterior cingulate cortex activity can be independent of response conflict in Stroop-like tasks. Proc Natl Acad Sci USA 103:13884-13889.

Rushworth MF, Walton ME, Kennerley SW, Bannerman DM (2004) Action 
sets and decisions in the medial frontal cortex. Trends Cogn Sci 8:410-417.

Sanders AF, Lamers JM (2002) The Eriksen flanker effect revisited. Acta Psychol (Amst) 109:41-56.

Sohn MH, Albert MV, Jung K, Carter CS, Anderson JR (2007) Anticipation of conflict monitoring in the anterior cingulate cortex and the prefrontal cortex. Proc Natl Acad Sci USA 104:10330-10334.

Somerville LH, Heatherton TF, Kelley WM (2006) Anterior cingulate cortex responds differentially to expectancy violation and social rejection. Nat Neurosci 9:1007-1008.

Swick D, Turken AU (2002) Dissociation between conflict detection and error monitoring in the human anterior cingulate cortex. Proc Natl Acad Sci USA 99:16354-16359.

Talairach J, Tournoux P (1988) Co-planar stereotaxic atlas of the human brain. Stuttgart, Germany: Thieme.

Toni I, Schluter ND, Josephs O, Friston K, Passingham RE (1999) Signal-, set- and movement-related activity in the human brain: an event-related fMRI study. Cereb Cortex 9:35-49.
Turken AU, Swick D (1999) Response selection in the human anterior cingulate cortex. Nat Neurosci 2:920-924.

Wallis JD, Miller EK (2003) From rule to response: neuronal processes in the premotor and prefrontal cortex. J Neurophysiol 90:1790-1806.

Walton ME, Bannerman DM, Alterescu K, Rushworth MF (2003) Functional specialization within medial frontal cortex of the anterior cingulate for evaluating effort-related decisions. J Neurosci 23:6475-6479.

Walton ME, Devlin JT, Rushworth MF (2004) Interactions between decision making and performance monitoring within prefrontal cortex. Nat Neurosci 7:1259-1265.

Weissman DH, Gopalakrishnan A, Hazlett CJ, Woldorff MG (2005) Dorsal anterior cingulate cortex resolves conflict from distracting stimuli by boosting attention toward relevant events. Cereb Cortex 15:229-237.

Williams ZM, Bush G, Rauch SL, Cosgrove GR, Eskandar EN (2004) Human anterior cingulate neurons and the integration of monetary reward with motor responses. Nat Neurosci 7:1370-1375.

Yeung N, Nystrom LE, Aronson JA, Cohen JD (2006) Between-task competition and cognitive control in task switching. J Neurosci 26:1429-1438. 